\title{
Results from Solar Reflective Band End-to-End Testing for VIIRS F1 Sensor Using T-SIRCUS
}

\author{
Jeff McIntire $^{\mathrm{a}}$, David Moyer ${ }^{\mathrm{b}}$, James K. McCarthyc ${ }^{\mathrm{c}}$, Frank De Luccia ${ }^{\mathrm{b}}$, \\ Xiaoxiong (Jack) Xiong ${ }^{d}$, James J. Butler ${ }^{d}$, and Bruce Guenther ${ }^{\mathrm{e}}$

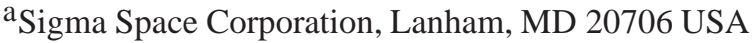 \\ jmcintire@sigmaspace.com

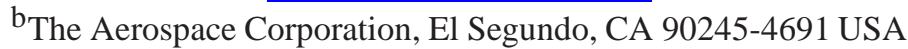 \\ ${ }^{\mathrm{c}}$ Northrop Grumman Aerospace Systems, Redondo Beach, CA 90278 USA

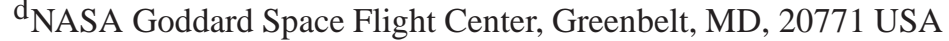 \\ ${ }^{\mathrm{e}}$ National Oceanic and Atmospheric Administration, Silver Spring, MD, 20910 USA
}

\begin{abstract}
Verification of the Visible Infrared Imager Radiometer Suite (VIIRS) End-to-End (E2E) sensor calibration is highly recommended before launch, to identify any anomalies and to improve our understanding of the sensor on-orbit calibration performance. E2E testing of the Reflective Solar Bands (RSB) calibration cycle was performed pre-launch for the VIIRS Fight 1 (F1) sensor at the Ball Aerospace facility in Boulder CO in March 2010. VIIRS reflective band calibration cycle is very similar to heritage sensor MODIS in that solar illumination, via a diffuser, is used to correct for temporal variations in the instrument responsivity. Monochromatic light from the NIST T-SIRCUS was used to illuminate both the Earth View (EV), via an integrating sphere, and the Solar Diffuser (SD) view, through a collimator. The collimator illumination was cycled through a series of angles intended to simulate the range of possible angles for which solar radiation will be incident on the solar attenuation screen on-orbit. Ideally, the measured instrument responsivity (defined here as the ratio of the detector response to the at-sensor radiance) should be the same whether the EV or SD view is illuminated. The ratio of the measured responsivities was determined at each collimator angle and wavelength. In addition, the Solar Diffuser Stability Monitor (SDSM), a ratioing radiometer designed to track the temporal variation in the SD BRF by direct comparison to solar radiation, was illuminated by the collimator. The measured SDSM ratio was compared to the predicted ratio. An uncertainty analysis was also performed on both the SD and SDSM calibrations.
\end{abstract}

Keywords: NPP VIIRS, pre-launch calibration

\section{INTRODUCTION}

VIIRS F1 sensor is a cross-track scanning radiometer developed for the NPP (NPOESS Preparatory Project) mission and is capable of making continuous global observations of the Earth intended for use in scientific studies of land, ocean, and atmosphere [1-3]. The design of VIIRS was heavily influenced by heritage sensor MODIS [4]. VIIRS RSB consists of 14 spectral bands covering a range of $\sim 0.4-2.3 \mu \mathrm{m}$ with resolutions at nadir of $375 \mathrm{~m}$ (3 bands) and $750 \mathrm{~m}$ (11 bands). In order to maintain the reflective band calibration on-orbit, VIIRS was equipped with three on-board calibration devices, the Solar Diffuser (SD), the Solar Attenuation Screen (SAS), and the Solar Diffuser Stability Monitor (SDSM).

VIIRS F1 sensor will rely on regular solar observations on-orbit for RSB calibration (similar to the heritage sensor MODIS [5]). These observations can occur once per orbit over the South pole for approximately three minutes. During these observations, solar illumination, scattered

Send correspondence to Jeff McIntire. 
off the $\mathrm{SD}$, will be used to correct for temporal variations in the instrument radiometric responsivity over the sensor lifetime. However, the SD is fabricated from space-grade Spectralon, the performance of which is known to degrade over time from exposure to the sun. The degradation of the SD BRF will be tracked by the SDSM, a ratioing radiometer which relates near simultaneous measurements of direct solar illumination to solar illumination diffusely reflected off the SD. It is important that the RSB on-orbit calibration cycle be performed during pre-launch testing, in order to identify any issues and/or anomalies as well as test the validity of the calibration cycle under controlled conditions.

E2E testing of the VIIRS F1 RSB calibration cycle was performed in March 2010 at the Ball Aerospace facility in Boulder CO. VIIRS had been successfully integrated into the NPP spacecraft two months earlier. The NIST T-SIRCUS (Traveling Spectral Irradiance and Radiance Responsivity Calibrations using Uniform Sources) [6] was transported to Boulder to act as a source for E2E testing (as well as spectral testing [7]). The T-SIRCUS was used to illuminate both the EV (through an integrating sphere) and SD view (via a collimator) using monochromatic light and was monitored in real-time. In addition, both paths into the SDSM were simultaneously illuminated.

E2E testing was not part of the baseline validation testing of VIIRS F1 sensor performed at the sensor subcontractor (Raytheon El Segundo). The E2E test was conducted during NPP spacecraft level testing to investigate whether this type of test setup could validate the VIIRS RSB calibration cycle pre-launch. Further, the use of tunable lasers in the calibration is in the exploratory phase. As such, this work is focused as much on the application of this technology to the calibration of VIIRS as to the results of the analysis.

This paper will compare the sensor responsivities derived from T-SIRCUS data for both views in order to assess the on-orbit SD calibration cycle as well as the SDSM calibration. Section 2 will review the design of the on-board RSB calibrators, Section 3 will provide a brief overview of the T-SIRCUS and a description of the test setup, Section 4 will establish the analysis methodology, and Section 5 will discuss the results of this analysis including the measurement uncertainty.

\section{RSB ON-BOARD CALIBRATOR OVERVIEW}

\subsection{Solar Diffuser and Solar Attenuation Screen}

VIIRS on-orbit calibration is reflectance based with reference to the SD composed of spacegrade Spectralon. The SD Bi-directional Reflectance Factor (BRF) was characterized prelaunch by the sensor subcontractor (then Raytheon SBRS) [8]. The BRF was measured at six wavelengths $(0.4,0.5,0.6,0.7,0.9$, and $1.7 \mu \mathrm{m})$, nine incident angles designed to replicate the range of possible on-orbit solar observations, and two angles of reflectance [corresponding to the Rotating Telescope Assembly (RTA) and SDSM views].

The SD views the sun through the SAS. Unlike MODIS, VIIRS SAS is fixed; consequently only one radiance level is available for on-orbit calibration per band. The vignetting function of the SAS was measured pre-launch by the sensor subcontractor [8]; at normal incidence, the transmission is $\sim 0.13$. In addition, no aperture door was incorporated into VIIRS design (in contrast, MODIS has an aperture door). As a result, the SD will be exposed to greater potential degradation of its BRF on-orbit than MODIS. Improvements in the design and prelaunch characterization of VIIRS (particularly with regard to the SDSM) will enable accurate tracking of this degradation.

\subsection{Solar Diffuser Stability Monitor}

Degradation in the SD BRF from $\sim 0.41-0.94 \mu m$ will be tracked on-orbit by the SDSM. The SDSM is a ratioing radiometer that compares direct solar illumination with light transmitted by the SAS and then diffusely reflected off the SD. The direct solar illumination is viewed through 
an attenuation screen, the transmission of which was characterized pre-launch by the sensor subcontractor (then Raytheon SBRS) [8]. The SDSM contains an integrating sphere embedded with eight detectors filtered to match VIIRS moderate resolution bands in the visible and near infrared (with the exception of the eighth detector at $935 \mathrm{~nm}$, which has no corresponding VIIRS band). A fold mirror directs light into this integrating sphere from one of three views: solar, SD, or dark (the dark view is used as a zero reference for the detectors). A stepping motor rotates this fold mirror through these positions every three scans repeatedly during an RSB calibration cycle.

The SDSM collects data during multiple on-orbit RSB calibrations over the sensor lifetime. A time-series of the ratios of the solar and SD signals will be used to track the SD BRF degradation, and to correct the responsivity on-orbit. Due to the fact that the same SDSM optics, detectors, and electronics are used for both SDSM solar and SD paths, any slow changes in their performance will not effect the quality of the SD BRF degradation monitoring.

\section{TEST OVERVIEW}

\subsection{T-SIRCUS}

NIST has developed a laser-based facility known as SIRCUS for use in radiance and irradiance responsivity calibrations [6]. A portable version known as T-SIRCUS (or Traveling SIRCUS) was transported to Ball Aerospace in Boulder CO during March 2010 for VIIRS spacecraft level testing. SIRCUS consists of a series of tunable lasers; for E2E testing, a Ti:sapphire laser was used in continuous wave mode to produce monochromatic illumination at 742 and $852 \mathrm{~nm}$ (and frequency doubling was used to reach $442 \mathrm{~nm}$ ).

The T-SIRCUS was used to feed two sources via fiber optics: an integrating sphere and a collimator. The integrating sphere was positioned in the EV at a scan angle of $\sim-0.2$ degrees (VIIRS nadir view is defined as 0 degrees). The sphere was approximately 30 inches in diameter with an exit aperture of about 10 inches. The sphere was mounted on a scissor jack with its exit aperture approximately $4 \mathrm{ft}$ from the telescope aperture at nadir. Two radiance monitors were used to track the radiance output from the sphere in real-time. The monitors were positioned just off the optical path, one above and one below.

The collimator was positioned to fully illuminate both the SD through the SAS and the solar entrance aperture of the SDSM. The exit aperture of the collimator was about 25 inches in diameter. The collimator was also mounted on a scissor jack, by which the angle of the incident light on the SD was varied. A total of seven collimator positions were performed covering five different angles, listed in Table 1 in the order performed. Note that the first, fourth, and fifth positions are repeated measurements of roughly the same angle. An irradiance monitor was positioned near the center of the collimator exit aperture, from which real-time tracking of the irradiance was performed.

\subsection{Collimator Uniformity}

The collimator beam was known to be spatially nonuniform. Different portions of the collimator beam illuminated the irradiance monitor, SD, and SDSM views. As a result, correction factors relating the collimator illumination at VIIRS SD view as well as the SDSM SD and solar views to the irradaince monitor view were calculated.

After most collimator positions, the collimator was repositioned to illuminate a large Spectralon panel at roughly the same angle as was incident on VIIRS. A CCD camera was used to photograph the Spectralon panel. From these images, the correction factor was determined. 
Table 1. Collimator positions measured during E2E testing. Angles are relative to VIIRS coordinate system.

\begin{tabular}{|c|c|c|c|c|}
\hline Position & Date & Declination & Azimuthal & Wavelengths (nm) \\
\hline 1 & $3 / 19 / 2010$ & 22.52 & 16.31 & 742,852 \\
\hline 2 & $3 / 19 / 2010$ & 13.64 & 16.87 & 742,852 \\
\hline 3 & $3 / 19 / 2010$ & 30.09 & 16.34 & 742,852 \\
\hline 4 & $3 / 19 / 2010$ & 22.37 & 16.44 & 742,852 \\
\hline 5 & $3 / 20 / 2010$ & 22.38 & 16.52 & 742,852 \\
\hline 6 & $3 / 20 / 2010$ & 30.44 & 15.21 & 742,852 \\
\hline 7 & $3 / 20 / 2010$ & 13.63 & 15.07 & $442,742,852$ \\
\hline
\end{tabular}

\subsection{Test Description}

For E2E testing, VIIRS was operated in diagnostic mode, during which 2048 unaggregated moderate resolution (750 $\mathrm{m}$ at nadir) EV samples were recorded covering scan angles from about -18.2 to 18.2 degrees. This corresponds to a subset of the full operational mode EV data. Each sample has an angular resolution of $\sim 0.017776$ degrees. The integrating sphere was positioned in the EV and fully illuminated samples between scan angles of $\sim-1.0$ to $\sim 0.7$ degrees. During the course of each scan, 48 moderate resolution samples were also recorded in each of the three calibration views: Space View (SV), SD, and On-Board Calibrator Blackbody (OBC BB). In addition, VIIRS was operated in fixed high gain mode, which restricts all dual gain bands to their low radiance settings.

VIIRS data for E2E testing was recorded in a three data collection cycle. During the first collection, the SD and SDSM were illuminated, while the EV was dark. For the second collection, the EV was illuminated, while the SD and SDSM views were dark. For the last collection, both the EV and SD views were dark. Each of these collections contained 128 VIIRS scans (roughly 3.8 minutes). For most collimator positions, this cycle was conducted twice per measured wavelength (except position 5 when it was conducted only once). Data collections were performed with the SIRCUS output at 442, 742, and $852 \mathrm{~nm}$ (442 nm was only measured once during collimator position 7); this corresponds to VIIRS bands M2, M6, and M7 (see Table 2).

In addition, during the first collection in each cycle, the SDSM mirror was rotating and data was recorded. The SDSM detectors corresponding to the bands measured are listed in Table 2.

Table 2. VIIRS bands (and corresponding SDSM detectors) used in E2E testing.

\begin{tabular}{|c|c|c|c|}
\hline Band & Center $\lambda(\mathrm{nm})$ & Bandwidth $(\mathrm{nm})$ & SDSM Detector \\
\hline M2 & 445 & 18 & 2 \\
\hline M6 & 746 & 15 & 6 \\
\hline M7 & 865 & 39 & 7 \\
\hline
\end{tabular}




\section{METHODOLOGY}

\subsection{SD Calibration}

In order to test the SD calibration cycle, the calculated responsivities (g) from both the EV and SD paths were compared, or

$$
\eta=\frac{g_{S D}}{g_{E V}} .
$$

Here the detector responsivity is defined as the ratio of the detector response (background subtracted digital response) to the at-sensor radiance. Ideally, the detector responsivities for the EV and SD paths should be equal. As a result, the calculated ratio will depend on how well the components in the optical path were characterized as well as knowledge of the input radiance (or irradiance).

The responsivity for the EV path is the detector response divided by the product of the integrating sphere radiance and the Response Versus Scan angle (RVS) at the Half Angle Mirror (HAM) Angle of Incidence (AOI) of the integrating sphere, or

$$
g_{E V}=\frac{d n_{E V}}{R V S_{E V} L_{E V}} .
$$

The RVS is the variation in instrument response to HAM AOI and was described in [9]. For this test, the EV HAM AOI was $\sim 36.3$ degrees. The EV radiance $\left(L_{E V}\right)$ was determined from the average radiance retrieved from the two monitors on the integrating sphere.

The responsivity for the SD path is the detector response divided by the product of the collimator irradiance, the SAS transmission, the BRDF of the SD at the RTA reflectance angle, the cosine of the projection angle onto the SD, and the RVS at the SD scan angle, or

$$
g_{S D}=\frac{d n_{S D}}{R V S_{S D} E_{S D} \tau_{S A S} B R D F_{R T A} \cos \left(\theta_{S D}\right)} .
$$

For VIIRS, the RVS has been normalized to the SD HAM AOI of $\sim 60.2$ degrees (i.e. $R V S_{S D}=$ 1). The SAS transmission is defined as [8]

$$
\tau_{S A S}(\delta, \phi)=0.1258[1-0.1538 \tan (\delta)][1-0.04746 \tan (\phi)]
$$

where $\delta$ and $\phi$ are the collimator declination and azimuthal angles, respectively. The reflectance off the SD is assumed to be Lambertian in the neighborhood of the RTA reflectance angle (i.e. $B R F_{R T A}=\pi B R D F_{R T A}$ ). The $\mathrm{BRF}$ was measured at component level testing for nine incident angles, two reflectance angles, and six wavelengths. The BRF was fit to the following function of declination and azimuthal angles for each measured wavelength and reflectance angle [10]:

$$
B R F(\delta, \phi, \lambda)=c_{0}(\lambda)+c_{1}(\lambda) \delta+c_{2}(\lambda) \phi+c_{3}(\lambda) \delta^{2}+c_{4}(\lambda) \phi^{2}+c_{5}(\lambda) \delta \phi
$$

The fit coefficients for the RTA reflectance angle are listed in Table 3. The final BRF is determined by interpolating Eq. (5) between wavelengths. The cosine of the projection angle $\theta_{S D}$ is the dot product of the sun vector and the SD normal vector [8], or

$$
\cos \left(\theta_{S D}\right)=\vec{n}_{\text {sun }} \cdot \vec{n}_{S D}
$$

where $\vec{n}_{S D} \equiv(0.29724,-0.21860,0.92944)$ and

$$
\vec{n}_{\text {sun }}=\frac{1}{\sqrt{1+\tan ^{2}(\delta)+\tan ^{2}(\phi)}}[1,-\tan (\phi), \tan (\delta)] .
$$


As discussed in Section 3.2, the collimator output is known to be spatially nonuniform. As a result, a correction factor $\left(\gamma_{1}\right)$ relating the collimator footprint on the SD to the irradiance monitor position is included, or

$$
E_{S D}=\gamma_{1} E_{m o n},
$$

where $E_{m o n}$ is the irradiance monitor output. The derivation of the collimator uniformity correction factor will be described in Section 4.3. As a result, Eq. (3) becomes

$$
g_{S D}=\frac{\pi d n_{S D}}{\gamma_{1} E_{m o n} \tau_{S A S} B R F_{R T A} \cos \left(\theta_{S D}\right)} .
$$

Table 3. SD BRF fit coefficients for RTA reflectance angle [10].

\begin{tabular}{|c|c|c|c|c|c|c|}
\hline Wavelength $(\mathrm{nm})$ & $c_{0}$ & $c_{1}$ & $c_{2}$ & $c_{3}$ & $c_{4}$ & $c_{5}$ \\
\hline 400 & 0.999406 & 0.000947 & -0.002517 & -0.000004 & -0.000010 & 0.000026 \\
\hline 500 & 1.008981 & 0.000454 & -0.002655 & 0.000004 & -0.000009 & 0.000032 \\
\hline 600 & 0.998804 & 0.001787 & -0.002700 & -0.000044 & -0.000009 & 0.000032 \\
\hline 700 & 0.966439 & 0.005746 & -1.002742 & -0.000157 & -0.000009 & 0.000032 \\
\hline 900 & 0.987594 & 0.002276 & -0.002797 & -0.000052 & -0.000011 & 0.000025 \\
\hline 1700 & 0.962194 & 0.005435 & -0.002978 & -0.000153 & -0.000011 & 0.000032 \\
\hline
\end{tabular}

Table 4. SD BRF fit coefficients for SDSM reflectance angle [10].

\begin{tabular}{|c|c|c|c|c|c|c|}
\hline Wavelength $(\mathrm{nm})$ & $c_{0}$ & $c_{1}$ & $c_{2}$ & $c_{3}$ & $c_{4}$ & $c_{5}$ \\
\hline 400 & 0.906011 & 0.005525 & -0.001012 & -0.000064 & -0.000020 & 0.000014 \\
\hline 500 & 0.976150 & -0.002411 & -0.001104 & 0.000161 & -0.000022 & 0.000013 \\
\hline 600 & 0.925660 & 0.003218 & -0.001126 & -0.000004 & -0.000022 & 0.000014 \\
\hline 700 & 0.921604 & 0.003537 & -0.001184 & -0.000008 & -0.000023 & 0.000013 \\
\hline 900 & 0.933213 & 0.000525 & -0.001686 & 0.000100 & -0.000024 & 0.000039 \\
\hline 1700 & 0.933563 & 0.000067 & -0.001312 & 0.000096 & -0.000027 & 0.000007 \\
\hline
\end{tabular}

\subsection{SDSM Calibration}

The measured SDSM ratio is defined as follows:

$$
R_{\text {measured }}=\frac{D N_{S D}-D N_{\text {dark }}}{D N_{\text {solar }}-D N_{\text {dark }}}
$$

The $R_{\text {measured }}$ is determined every three scans and is trended over a particular calibration cycle as well as across calibration cycles. This allows for tracking of the temporal changes in the SD BRF.

The irradiance reaching the SDSM integrating sphere aperture using the SD path is

$$
E_{S D S M-S D}=\gamma_{2} E_{m o n} \tau_{S A S} \frac{B R F_{S D S M}}{\pi} \cos \left(\theta_{S D}\right) \pi \sin ^{2}(\psi),
$$


where $\tau_{S A S}$ and $\cos \left(\theta_{S D}\right)$ are defined in Eqs. (4) and (6), respectively. The SD BRF is described by Eq. (5) using the fit coefficients for the SDSM reflectance angle listed in Table 4. The factor of $\pi \sin ^{2}(\psi)$ corresponds to the solid angle of the entrance cone on the SDSM for the SD view, where the half angle of the cone, $\psi$, is 7.78 degrees [8]. $\gamma_{2} E_{m o n}$ is the measured irradiance of the collimator (including uniformity correction factor $\gamma_{2}$ ).

The irradiance illuminating the SDSM integrating sphere aperture using the solar path is

$$
E_{S D S M-\text { solar }}=\gamma_{3} E_{m o n} \tau_{S D S M}
$$

$\tau_{S D S M}$ is the transmission factor for the SDSM screen. This transmission includes the cosine of the projection angle onto the SDSM screen. In addition, because the detectors are located inside the SDSM integrating sphere, each detector has a direct view of the reflection of the image on the inside of the sphere. This results in each detector observing a slightly different radiance based on its location inside the integrating sphere [8]. Note that the collimator uniformity correction for the SDSM SD view is different from the correction for the solar view of the SDSM.

The theoretical SDSM ratio is the ratio of Eqs. (11) and (12), or

$$
R_{\text {calculated }}=\frac{\gamma_{2}}{\gamma_{3}} \frac{\tau_{S A S}}{\tau_{S D S M}} \cos \left(\theta_{S D}\right) B R F_{S D S M} \sin ^{2}(\psi) .
$$

The source irradiance cancels (excepting the uniformity corrections) and what remains is the ratio of the transmission factors in each optical path. Note that the transfer function from the SDSM integrating sphere aperture to the SDSM detector is common to both paths and would also cancel in the ratio.

\subsection{Collimator Uniformity Correction}

Table 5. Collimator uniformity correction for the SD calibration versus collimator position.

\begin{tabular}{|c|c|c|c|c|c|c|c|}
\hline Wavelength $(\mathrm{nm})$ & 1 & 2 & 3 & 4 & 5 & 6 & 7 \\
\hline 742 & 0.967 & 0.965 & 0.966 & 0.964 & 0.958 & 0.959 & 0.959 \\
\hline 852 & 0.940 & 0.937 & 0.933 & 0.936 & 0.924 & 0.926 & 0.933 \\
\hline
\end{tabular}

\subsection{Uncertainty Analysis}

The following uncertainty analysis uses the standard error propagation to combine the individual uncertainties involved in the present experiment to a total uncertainty [11].

The measurement error in the EV responsivity is given by the standard error propagation, or

$$
\frac{u^{2}\left(g_{E V}\right)}{g_{E V}^{2}}=\frac{u^{2}\left(d n_{E V}\right)}{d n_{E V}^{2}}+\frac{u^{2}\left(R V S_{E V}\right)}{R V S_{E V}^{2}}+\frac{u^{2}\left(L_{E V}\right)}{L_{E V}^{2}} .
$$

The SD responsivity uncertainty is also given by the standard error propagation, or

$$
\begin{aligned}
\frac{u^{2}\left(g_{S D}\right)}{g_{S D}^{2}}= & \frac{u^{2}\left(d n_{S D}\right)}{d n_{S D}^{2}}+\frac{u^{2}\left(R V S_{S D}\right)}{R V S_{S D}^{2}}+\frac{u^{2}\left(\gamma_{1}\right)}{\gamma_{1}^{2}}+\frac{u^{2}\left(E_{S D}\right)}{E_{S D}^{2}} \\
& +\frac{u^{2}\left(\tau_{S A S}\right)}{\tau_{S A S}^{2}}+\frac{u^{2}\left(B R F_{R T A}\right)}{B R F_{R T A}^{2}}+\frac{u^{2}\left[\cos \left(\theta_{S D}\right)\right]}{\cos ^{2}\left(\theta_{S D}\right)}
\end{aligned}
$$


Since most of these factors were measured independently $\left[\tau_{S A S}, B R F_{R T A}, L_{E V}, E_{S D}\right.$, and $\left.\cos \left(\theta_{S D}\right)\right]$, it is assumed that these factors have no correlations with any other factor. In addition, although $d n_{S D}, d n_{E V}, R V S_{E V}$, and $R V S_{S D}$ were all measured using VIIRS detectors, they were measured separately and therefore are also assumed to be uncorrelated with the other error sources. The measurement error in the responsivity ratio is then

$$
\frac{u^{2}(\eta)}{\eta^{2}}=\frac{u^{2}\left(g_{S D}\right)}{g_{S D}^{2}}+\frac{u^{2}\left(g_{E V}\right)}{g_{E V}^{2}}
$$

The error in the calculated SDSM ratio is

$$
\begin{aligned}
\frac{u^{2}\left(R_{\text {calculated }}\right)}{R_{\text {calculated }}^{2}}= & \frac{u^{2}\left(\tau_{S A S}\right)}{\tau_{S A S}^{2}}+\frac{u^{2}\left(\tau_{S D S M}\right)}{\tau_{S D S M}^{2}}+\frac{u^{2}\left[\cos \left(\theta_{S D}\right)\right]}{\cos ^{2}\left(\theta_{S D}\right)} \\
& +\frac{u^{2}\left(B R F_{S D S M}\right)}{B R F_{S D S M}^{2}}+4 \frac{u^{2}[\sin (\psi)]}{\sin ^{2}(\psi)}+\frac{u^{2}\left(\gamma_{2}\right)}{\gamma_{2}^{2}}+\frac{u^{2}\left(\gamma_{3}\right)}{\gamma_{3}^{2}} .
\end{aligned}
$$

Since all of these factors in $R_{\text {calculated }}$ were measured independently, it is assumed that there are no correlations between the uncertainties of the various factors.

\section{ANALYSIS RESULTS}

\subsection{SD Calibration}

\subsubsection{Data Reduction}

The integrating sphere aperture covered an arc of about 2 deg rees of scan angle centered roughly on nadir. From this region, 90 moderate resolution $(750 \mathrm{~m}$ ) samples were extracted (samples 970-1059). These 90 samples were averaged for every scan (ideally there are 128 scans per data collection) and for each VIIRS detector (16 detectors per VIIRS moderate resolution band). In addition, the 48 samples extracted from both the SD and OBC BB views were similarly averaged (note that calibration view data is reported in 14 bits while EV data is automatically truncated from 14 to 12 bits by VIIRS; in this analysis all calibration view data is first truncated to 12 bits to remove any potential bias). For each average, those points more than three standard deviations from the mean were excluded. The OBC BB was maintained at $\sim 292 \mathrm{~K}$ throughout the testing; as a result, the OBC BB data was used as a zero reference for both the EV and SD data. The resulting scan dependent $d n_{E V}$ and $d n_{S D}$ were substituted into Eqs. (2) and (9), respectively.

The integrating sphere radiance and collimator irradiance were tracked in real-time by monitors (two monitors on the sphere and one monitor on the collimator). The output of each monitor was provided in volts, and was converted to either radiance or irradiance (the average of the two radiance monitors is used here) using predetermined monitor calibration coefficients provided by NIST. Output from each monitor was obtained about every $10 \mathrm{~s}$. Linear interpolation was used to acquire the radiance or irradiance at the particular VIIRS time stamp associated with each scan. The resulting scan dependent $L_{E V}$ and $E_{S D}$ were input into Eqs. (2) and (9), respectively.

Using Eqs. (2) and (9), the EV and SD responsivities were determined on a scan by scan basis. Since the EV and SD data was recorded at separate times, the scan averaged EV and SD responsivities were calculated for each data collection. These scan averaged responsivities were then substituted into Eq. (1), from which the responsivity ratio was determined for each three data collection cycle. The average of the two collection cycles per collimator position was then calculated.

The following sections will only discuss the results for HAM side A, but both HAM sides yield consistent results. 


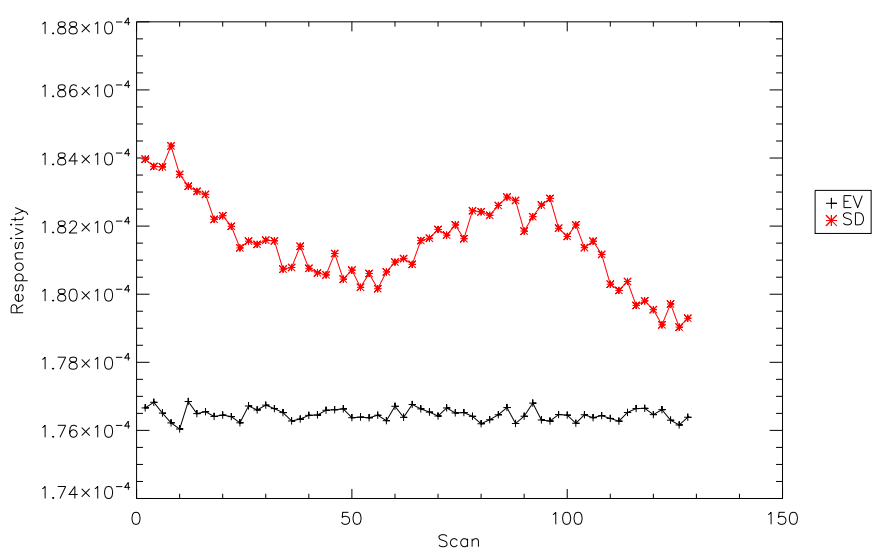

Fig. 1. M6 scan dependent responsivities (both EV and SD) from collimator position 7, measurement 2.

\subsubsection{Data Quality}

A number of data quality checks were performed during the processing of the data for the SD calibration. Listed below are the major findings of those checks.

Some of the data collections did not contain the full expected 128 scans of VIIRS data (collections at positions 3 and 5, both at $852 \mathrm{~nm}$ ). For those collections, the data was retained for those scans that appeared to be valid data (i.e. they were consistent with the data collections in which all scans were present).

The signal observed in the SD view when the EV was illuminated via the integrating sphere was negligible; in contrast, the response reported in the EV when the SD was illuminated via the collimator was between $2-3$ dn for most cases. This is the possible result of scatter from the collimator reaching the EV, and as such has no real effect on the calibration. In addition, the data from the dark collections was analyzed and the resulting dn was negligible for all cases.

The standard deviation of the EV dn was determined over scans at each sample and also over samples at each scan (similarly for the SD dn). For the SD view, the two methods were comparable. However, for the EV, the standard deviation over samples was lower than the standard deviation over scans. This indicates that the integrating sphere had higher spatial stability than temporal stability (and that the measured SNR was closer to the true sensor SNR using the first method).

The stability of the sources was also tracked via their respective monitors. The two monitors on the integrating sphere show some variation over time (they are both individually stable to within about $1.1 \%$ at $742 \mathrm{~nm}$ and $2.1 \%$ at $852 \mathrm{~nm}$ ); in addition, the two monitors vary in approximately the same manner. However, the monitors are offset from each other by about 2 $\%$ for $742 \mathrm{~nm}$ and $0.2 \%$ for $852 \mathrm{~nm}$. The collimator monitor recorded temporal variation of up to about $2.9 \%(3.3 \%)$ at $742 \mathrm{~nm}(852 \mathrm{~nm})$ over a particular data collection.

The EV dn and monitor radiances have similar temporal trends, and as such the calculated $\mathrm{EV}$ responsivity at each scan is generally consistent over time. In contrast, the SD dn and collimator irradiance have different temporal dependencies, with the result being that the calculated SD responsivity is not necessarily constant over scans; an example of this behavior is plotted in Figure 1 which shows the scan by scan EV and SD responsivities for M6 collimator position 7 (second data collection). The inability of the collimator monitor to effectively track the incident irradiance is a major source of uncertainty for the SD responsivity. 


\subsubsection{SD Uncertainty Analysis}

The error propagation for the SD calibration was defined by Eq. (16). The individual uncertainties that are used in Eq. (16) are described in this section. In general, each uncertainty contributor is the combination of precision (random) and accuracy (biases) errors.

The error in the SD and EV dn is a combination of the precision and accuracy uncertainties. The precision error is the standard deviation of the mean over all samples and scans in a given data collection. The accuracy uncertainty accounts for any biases between the SD or EV and the $\mathrm{OBC}$ views (since any biases common to all sectors cancel in the background subtraction). The known biases between sectors are either accounted for in the processing (truncation of calibration views to 12 bits before processing begins) or not applicable (biases due to aggregation or auto/fixed gain differences); as a result, no accuracy uncertainty is included here.

The RVS uncertainties were determined during ambient phase testing at the instrument level. The band average values used in this work are 0.000570 [M6] and 0.000558 [M7], and are used for both SD and EV angles. These values are the Root Sum Square (RSS) of fitting and measurement uncertainties [9]. The uncertainties associated with the EV radiance are the combination of precision and accuracy uncertainties. The precision error is determined by calculating the standard deviation of the mean over all radiance monitor values for a particular data collection (both monitors). The accuracy error is the average offset between the two radiance monitors over a data collection. The total radiance uncertainty is the RSS of the precision and accuracy errors. The error in the SD irradiance is the standard deviation of the mean over all irradiance monitor values in each data collection (precision error). The accuracy error was undetermined. The error in the SAS transmission and the SD BRF were determined by the sensor subcontractor [8], and are $0.24 \%$ and $1.09 \%$, respectively. The cosine of the projection angle is determined from alignment measurements made using theodolites and Eq. (6). The measurements are assumed to be known to 0.01 degrees. That uncertainty is propagated through Eq. (6). The uncertainty in the collimator uniformity correction is currently in progress and is left for future work. The final responsivity uncertainties are determined by Eqs. (14) and (15). Then, the uncertainty in the responsivity ratio at a particular data collection cycle is determined from Eq. (16).

\subsubsection{SD Calibration Results}

The derived M6 and M7 responsivity ratios for each detector and collimator position are shown in Figure 2. In the case of M6, all collimator positions are within $\sim 2 \%$ of one. In addition, the responsivity ratio tends to increase over detectors for all collimator positions (particulary positions 1 and 7). For M7, all positions are within $\sim 4 \%$ of unity, with the exception of position 7 which is between 4 and $6 \%$ below one. Here the detector variation is again roughly increasing, but more uniformly over collimator position than for M6. This detector trend likely results from detector dependence in the collimator uniformity correction (the RTA footprint on the SD is slightly different for each detector and band, which in turn requires a slightly different collimator correction). Although E2E testing was also conducted at $442 \mathrm{~nm}$ (M2) for collimator postion 7 , the digital response was too low to accurately determine the responsivity ratio $\left(d n_{S D} \sim 6\right.$ and $\left.d n_{E V} \sim 15\right)$.

Measurement repeatability was investigated both on short time scales (minutes) and long time scales (hours). The collimator was positioned three times at roughly the same angle (positions 1, 4, and 5 in Table 1). The interval between positions 1 and 4 was about 6 hours and the interval between positions 4 and 5 was about 16 hours. For M6 (see the black, green, and yellow curves in the upper plot of Figure 2), the ratios are within $1.0 \%$ of unity for all detectors for positions 4 and 5; however, the ratios derived from position 1 are lower (between 0.5 and 2 $\%$ below one). In addition, the detector variation is greater for position 1. In contrast, M7 shows the ratios derived from positions 1 and 5 in good agreement with each other (although between 

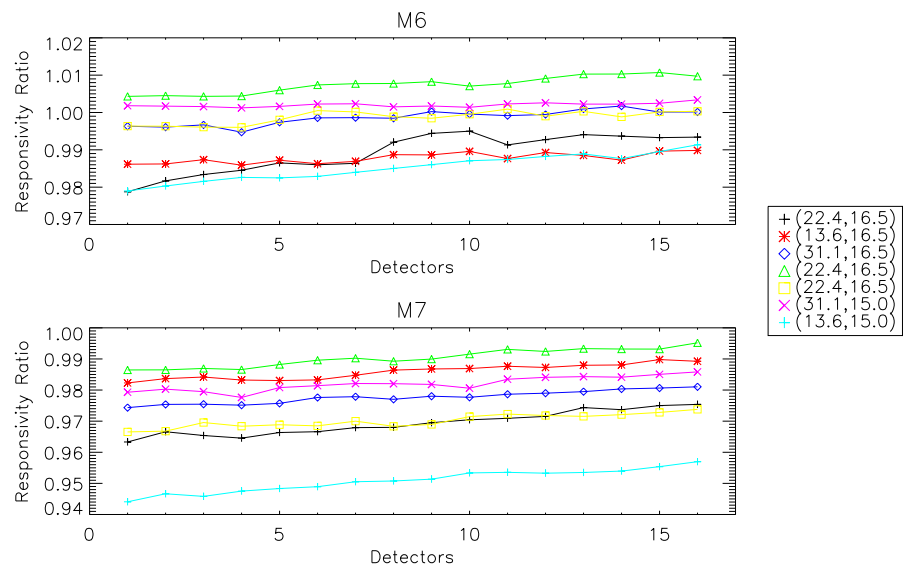

Fig. 2. M6 and M7 responsivity ratios versus VIIRS detector. Collimator positions are given in (declination angle, azimuthal angle).

2 and $4 \%$ below one), while the ratios from position 4 are between 0.5 and $1.5 \%$ below one (see the black, green, and yellow curves in the lower plot of Figure 2).

In terms of short term stability, the E2E test was repeated for each collimator position twice within about 30 minutes (except position 5). The data from each individual collection cycle (SD, EV, dark) was used to derive a responsivity ratio. The comparison of the short term stability is shown in Figure 3 versus detector. Here the solid (dashed) lines indicate the first (second) measurement. For M6, positions 4 and 7 agree well, while positions 1, 2, 3, and 6 show discrepancies up to $1 \%$. The ratios deteremined for $\mathrm{M} 7$ show agreement to within $0.5 \%$ for all positions except postion 2 , for which short term repeatablilty is about $2 \%$.

The discrepancies between the repeated measurements are largely understood by examining the underlying $\mathrm{dn}$, radiance, and irradiance. In Table 6 , the $\mathrm{dn}$, radiance, and irradiance are listed for M6 detector 9 at each collimator position and measurement repeat (averaged over scans). For the EV, the dn increases when the radiance increases; however, this is not always the case for the SD measurements. Collimator positions 1, 2, and 7 actually have lower irradiance for the measurement with higher $\mathrm{dn}$. This result also holds when examining the SD on a scan basis. Thus the discrepancies in the repeatability measurements appear to be largely related to the accuracy of the irradiance monitor output.

The band averaged uncertainty estimates based on Eq. (16) are listed in Table 7 for all collimator positions. The BRF uncertainty is the largest contributor to the uncertainty for M7 (at $1.09 \%$ ). For M6, the largest contributor is the accuracy error for the EV radiance, which is around $1.7 \%$, follwed by the BRF uncertainty. Because the main sources of error were not detector dependent, the varition of the uncertainty with detector is small for both bands. Note that the collimator uniformity correction uncertainty was not included; it is expected that this uncertainty will be a major contributor.

Figure 4 shows the band averaged responsivity ratio for each collimator position with the uncertainties shown in Table 7. The responsivity ratios are consistent with one for band M6 for all collimator positions. However, this is not the case for band M7, where only collimator position 4 is consistent with one; all other positions are lower (especially position 7 which is much lower). 

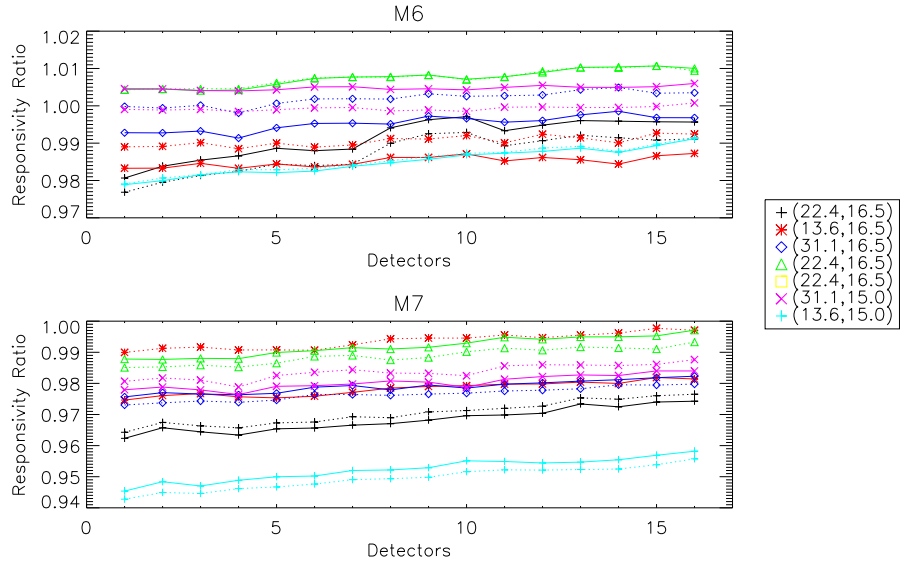

Fig. 3. M6 and M7 resposnsivity ratios - short term stability. Collimator positions are given in (declination angle, azimuthal angle).
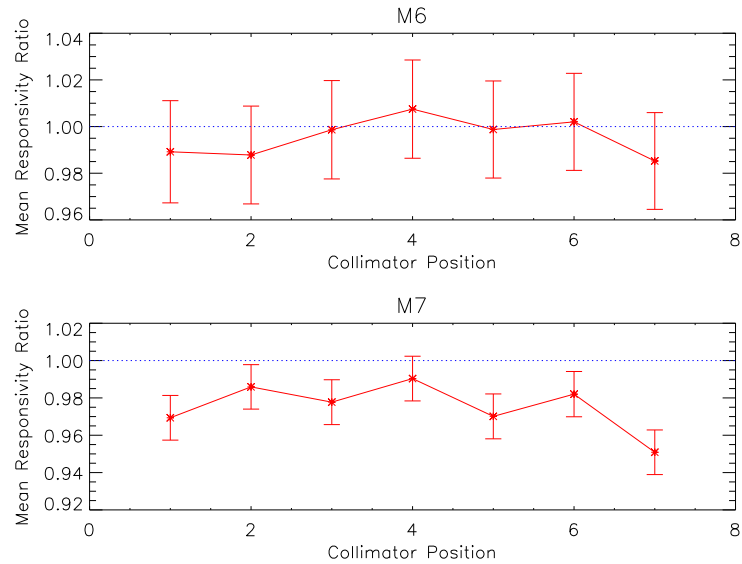

Fig. 4. Band averaged responsivity ratios with uncertainties. 
Table 6. Short term repeatability for M6 detector 9 (HAM side A). dn, radiance, and irradiance are averaged over scans.

\begin{tabular}{|c|c|c|c|c|c|c|}
\hline Position & Measurement & $E_{S D}$ & $d n_{S D}$ & $L_{E V}$ & $d n_{E V}$ & $\eta$ \\
\hline 1 & 1 & 4.728 & 297.7 & 0.1062 & 292.7 & 0.996 \\
\hline 1 & 2 & 4.735 & 297.4 & 0.1061 & 292.7 & 0.993 \\
\hline 2 & 1 & 4.701 & 286.2 & 0.1071 & 295.2 & 0.986 \\
\hline 2 & 2 & 4.729 & 283.7 & 0.1061 & 292.7 & 0.991 \\
\hline 3 & 1 & 4.674 & 290.2 & 0.1053 & 290.6 & 0.997 \\
\hline 3 & 2 & 4.612 & 287.6 & 0.1055 & 291.3 & 1.003 \\
\hline 4 & 1 & 4.747 & 291.2 & 0.1076 & 296.7 & 1.008 \\
\hline 4 & 2 & 4.735 & 291.4 & 0.1069 & 294.8 & 1.008 \\
\hline 6 & 1 & 5.924 & 354.6 & 0.1286 & 355.2 & 1.005 \\
\hline 6 & 2 & 6.006 & 358.1 & 0.1319 & 364.1 & 0.999 \\
\hline 7 & 1 & 6.089 & 369.1 & 0.1286 & 354.4 & 0.986 \\
\hline 7 & 2 & 6.084 & 369.9 & 0.1278 & 351.9 & 0.986 \\
\hline
\end{tabular}

Table 7. Responsivity ratio uncertainties in percent.

\begin{tabular}{|c|c|c|c|c|c|c|c|}
\hline Uncertainty & 1 & 2 & 3 & 4 & 5 & 6 & 7 \\
\hline $\mathrm{M} 6 u(\eta)$ & 2.19 & 2.10 & 2.11 & 2.11 & 2.08 & 2.08 & 2.07 \\
\hline $\mathrm{M} 7 u(\eta)$ & 1.20 & 1.19 & 1.20 & 1.20 & 1.20 & 1.21 & 1.20 \\
\hline
\end{tabular}

\subsection{SDSM Calibration}

\subsubsection{Data Quality and Reduction}

Five SDSM samples were recorded every scan for each detector in the SDSM integrating sphere. These five samples were averaged for every scan. The averaged DN from each three scan cycle was used to determine the ratio in Eq. (10). This SDSM ratio was trended versus time for each data collection and collimator angle. In addition, the average of the ratios for each data collection was determined. As the SDSM was illuminated with monochromatic light, only the detector corresponding to the input wavelength was analyzed. As with the SD data, some data collections were missing scans; again, the data was retained for those scans that appeared to be valid data. The collimator uniformity corrections $\left(\gamma_{2}\right.$ and $\left.\gamma_{3}\right)$ were not applied in this work.

\subsubsection{SDSM Uncertainty Analysis}

The error propagation for the SDSM calibration was defined in Section 4.4. The uncertainties of the measured SDSM ratio as well as the individual uncertainties that are used in the calculated SDSM error are described in this section, along with the total uncertainty in the calculated SDSM ratio.

Due to the large variability in the SDSM measurement over any given data collection (as shown in Figure 5), the error on the average SDSM ratio per data collection is taken to be the standard deviation of all individual SDSM ratios within that data collection. 
The error in the SAS transmission, the SDSM screen transmission, and the SDSM BRF were determined by the sensor subcontractor [8], and are $0.24 \%, 0.67 \%$, and $1.09 \%$, respectively. The uncertainty in the cosine of the projection angle is determined from alignment measurements made using theodolites. The measurements are assumed to be known to 0.01 degrees. That uncertainty is propagated through Eq. (6). The SDSM entrance cone angle is also assumed to be known to 0.01 degrees; the uncertainty is propagated to the solid angle of the entrance cone. The determination of the uncertainties for the collimator uniformity correction factors is in progress; for the purposes of this work, they are not included in the final results presented below. The final calculated SDSM ratio uncertainties are defined by Eq. (17) for each data collection.

\subsubsection{Results}

The measured SDSM ratios [as defined by Eq. (10)] are plotted in Figure 5. Each color represents a different collimator position (solid and dashed lines indicating first and second data collections at a particular collimator position). The ratios are between 1.1 and 1.3 for $742 \mathrm{~nm}$ and between 1.15 and 1.35 for $852 \mathrm{~nm}$. In addition, the measured ratios fluctuate by up to 5 $\%$ over roughly 3.8 minutes (the variaition in the solar view is generally larger than in the SD view). On MODIS, the SDSM also exhibits large variation in any given detector over a typical solar observation; fortunately, all the SDSM detectors vary in roughly the same manner. This allows the temporal variation to be removed using the response of MODIS SDSM detector 9 $(936 \mathrm{~nm})$. Due to the fact that T-SIRCUS was a narrow band source, only one SDSM detector recoded a meaningful signal for a particular data collection; as a result, the temporal trend could not be removed in this test using the MODIS methodology.

The measured ratios, averaged over each data collection, are plotted versus collimator position number in Figure 6 for both $742 \mathrm{~nm}$ (upper plot) and $852 \mathrm{~nm}$ (lower plot). Black and red points indicate the first or second data collections at a particular collimator position (where the error bars are \pm one standard deviation over that data collection). The blue curve represents the calculated ratio at each collimator position. For M6, the calculated and measured ratios agree well for all collimator positions. For M7, there is some disagreement between the theoretical and measured values as well as some discrepancy between the first and second data collections at some collimator positions.

The measured SDSM uncertainty as defined above was between roughly 0.5 and $2.1 \%$. The calculated error was approximately $1.38 \%$ (this number was fairly constant as the major factors were not wavelength or angle dependent). The largest contributor was the SD BRF (1.09\%). The measurements are consistent with the theoretical values for all positions at $742 \mathrm{~nm}$ (with the exception of position 3, measurment 1). However, most measurements are not consistent with the theoretical results at $852 \mathrm{~nm}$.

\section{CONCLUSIONS}

E2E testing of a limited number of VIIRS F1 reflective solar bands was conducted in March 2010 at Ball Aerospace in Boulder CO using the NIST T-SIRCUS. The E2E test was an exploration of the use of tunable lasers in the validation of the RSB calibration cycle pre-launch. The goal of the testing was to verify that the responsivites determined from the EV and SD paths were consistent. In addition, the SDSM measurements were compared to the modeled ratios.

The EV / SD responsivity ratios were consistent with one for band M6, but inconsistent for band M7. Slight upward trending with detector was observed in the responsisvity ratios, which likely results from detector dependence of the collimator uniformity correction. The SDSM measured and theoretical values are consistent at $742 \mathrm{~nm}$, but inconsistent at $852 \mathrm{~nm}$. In addition, the SDSM ratios exhibit some temporal variability. 

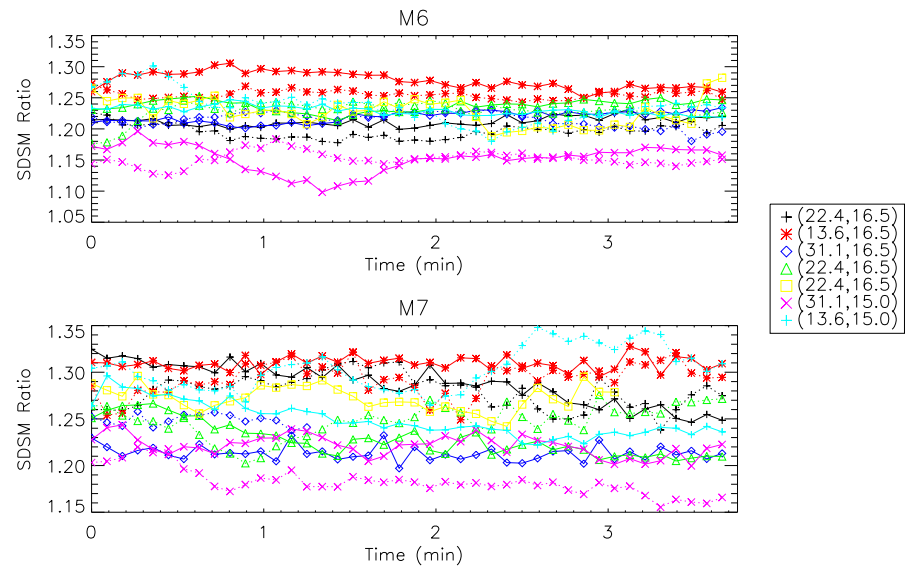

Fig. 5. M6 and M7 SDSM measured ratios - short term stability.

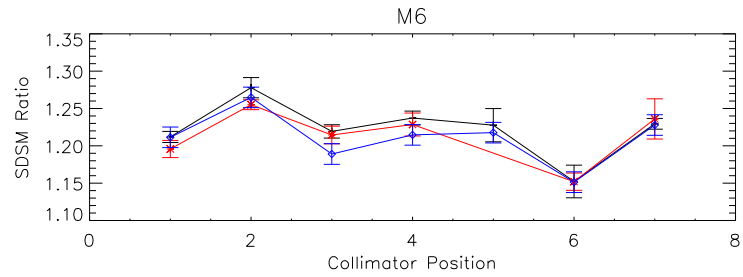

M7

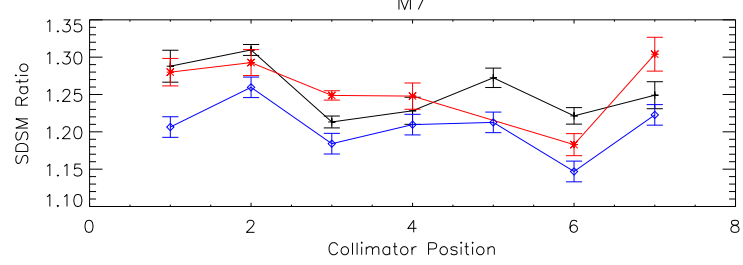

Fig. 6. M6 and M7 SDSM ratios (measured and calculated) with uncertainty estimates. 
A large source of uncertainty is in the collimator irradiance, which in turn is heavily dependent on the collimator uniformity correction. This correction is very complicated and is still in progress. It is expected that an updated correction will improve the agreement of the responsivity ratio for M7 as well as decrease the detector dependence. In addition, uniformity corrections in the SDSM analysis have yet to be determined, which it is hoped will improve agreement for the SDSM ratios at $852 \mathrm{~nm}$.

The E2E test was a preliminary rough validation of the RSB calibration cycle. Improvements in the test setup should reduce the uncertainties and improve the accuracy of the measurement (particularly with respect to the collimator irradiance). Future applications of this test methodology should expect to accurately validate VIIRS RSB calibration cycle pre-launch.

\section{Acknowledgments}

The authors would like to acknowledge the following: Steve Brown, Keith Lykke, and Mike Zhu from NIST who operated T-SIRCUS in Boulder CO and provided many useful insights into the calibration; Hassan Oudrari for his helpful comments on the methodology and analysis; members of NICST and NICSE who reviewed the preliminary analysis and provided comments; and the test team at Ball Aerospace for conducting the test.

\section{References}

[1] C. McClain, S. Hooker, G. Feldman, and P. Bontempi, "Satellite data for ocean biology, biogeochemistry, and climate research," Eos 87(34), 337-339 (2006).

[2] M. D. King, W. P. Menzel, Y. J. Kaufman, D. Tanre, B.-C. Gao, S. Platnick, S. A. Ackerman, L. A. Remer, R. Pincus, and P. A. Hubanks, "Cloud and aerosol and water vapor properties, precipitable water, and profiles of temperature and humidity from MODIS," IEEE Trans. Geosci. Remote Sen. 41, 442-458 (2003).

[3] C. O. Justice, E. Vermote, J. R. G. Townshend, R. Defries, D. P. Roy, D. K. Hall, V. V. Salomonson, J. L. Privette, G. Riggs, A. Strahler, W. Lucht, R. B. Mynemi, P. Lewis, and M. J. Barnsley, "The moderate resolution imaging spectroradiometer (MODIS): land remote sensing for global change research," IEEE Trans. Geosci. Remote Sen. 36, 1228 1249 (1998).

[4] W. L. Barnes and V. V. Salomonson, "A global image spectroradiometer for the earth observing system," Crit. Rev. Opt. Sci. Technol. , 285-307 (1993).

[5] X. Xiong, J. Sun, W. Barnes, V. Salomonson, J. Esposito, H. Erives, and B. Guenther, "Multiyear on-orbit calibration and performance of terra MODIS reflective solar bands," IEEE Trans. Geosci. Remote Sen. 45(4), 879-889 (2007).

[6] S. W. Brown, G. P. Eppeldauer, and K. R. Lykke, "Facility for spectral irradiance and radiance responsivity calibrations using uniform sources," Appl. Opt. 45(32), 8218-8237 (2006).

[7] R. A. Barnes, S. W. Brown, K. R. Lykke, B. Guenther, X. Xiong, and J. J. Butler, "Comparison of two methodologies for calibrating satellite instruments in the visible and near infrared," Proc. of SPIE 7862 (2010).

[8] B. Robinson, M. Bliton, R. Menzel, and D. Tyler, "Performance verification report VIIRS FU1 reflective band calibration (PVP section 4.2.2)," tech. rep., Raytheon (2009).

[9] C. Pan and N. Che, "Drift corrected VIIRS FU1 response versus scan (RVS) for the reflective solar bands (RSB) from FP-10 test." NICST Technical Memo 7 (2008).

[10] J. Sun and S. Xiong, "VIIRS FU1 SD prelaunch BRF for both VIIRS and SDSM view." NICST Technical Memo 43 (2008).

[11] J. R. Taylor, An Introduction to Error Analysis, University Science Books (1997). 